\title{
Representações sociais, conhecimento científico e fontes de informação sobre aids ${ }^{1}$
}

\author{
Jean Carlos Natividade ${ }^{2}$ \\ Universidade Federal do Rio Grande do Sul, Porto Alegre-RS, Brasil \\ Brígido Vizeu Camargo \\ Universidade Federal de Santa Catarina, Florianópolis-SC, Brasil
}

\begin{abstract}
Resumo: Investigaram-se, neste estudo, as relações entre a valorização simbólica atribuída a onze elementos descritores da representação social da aids, o nível de conhecimento científico sobre o HIV e aids, e as fontes de obtenção de informações sobre aids. Utilizou-se um questionário autoaplicável, de respostas fechadas, em 150 estudantes do ensino médio de uma escola pública. Identificaram-se relações significativas entre os elementos, que apontaram três fatores como caracterizadores da representação social da aids: responsabilidade diante do contágio; medo das consequências do contágio; descrição da aids. Foram encontradas relações significativas entre a valorização simbólica de seis elementos e quatro fontes de informação, que corroboraram a importância da comunicação extragrupo na constituição das representações sociais. Contudo, entre a valorização simbólica dos elementos e os níveis de conhecimento científico as relações não se mostraram significativas.
\end{abstract}

Palavras-chave: representação social, aids, adolescentes.

\section{Social representations, scientific knowledge and sources of information about AIDS}

\begin{abstract}
This study investigated the relationships among the symbolic valuation given to eleven descriptor elements of the social representation of AIDS, the level of scientific knowledge about HIV and AIDS, and the information sources about aids. A self-administered questionnaire with closed-ended questions was employed in 150 high school students from a public school. Significant relationships between the elements were identified, that indicated three characterizing factors of the social representation of AIDS: responsibility towards contagion, fear of contagion consequences and aids description. There were also relationships between the symbolic valuation of six elements and four information sources, which corroborated the importance of communication outside the group in the constitution of social representations. However, the relationships between the symbolic valuation of elements and the levels of scientific knowledge were non-significant.
\end{abstract}

Keywords: social representation, acquired immune deficiency syndrome, adolescents.

\section{Las representaciones sociales, el conocimiento científico y las fuentes de información sobre el SIDA}

\begin{abstract}
Resumen: Este estudio investigó la relación entre el valor simbólico atribuido a once elementos descriptores de la representación social del SIDA, el nivel de conocimientos científicos sobre el VIH y el SIDA, y las fuentes para obtener información sobre el SIDA. Se utilizó un cuestionario autoadministrado con respuestas cerradas en 150 estudiantes de secundaria en una escuela pública. Se identificaron relaciones significativas entre los elementos, que señaló tres factores que caracterizan la representación social del SIDA: la responsabilidad ante el contagio, el temor de las consecuencias de la infección, la descripción del SIDA. Y relaciones entre el valor simbólico de seis elementos y cuatro fuentes de información, que confirmó la importancia de la comunicación fuera del grupo en las representaciones sociales. Sin embargo, entre el valor simbólico de los elementos y niveles de conocimiento científico las relaciones no fueron significativas.
\end{abstract}

Palabras clave: representación social, síndrome de inmunodeficiencia adquirida, adolescente.

A aids é uma doença descrita pela ciência há menos de 30 anos. Embora se estime que seu agente etiológico - o vírus HIV (Human Immunodeficiency Virus) - esteja em contato com humanos há 100 anos (Worobey e cols., 2008), foi

\footnotetext{
${ }^{1}$ Este texto foi revisado seguindo o Acordo Ortográfico da Língua Portuguesa (1990), em vigor a partir de $1^{\circ}$ de janeiro de 2009. Os autores agradecem a João Fernando Rech Wachelke por apontamentos feitos durante a elaboração do instrumento de coleta e na análise de dados e a Gabriela Dal Forno Martins por sugestões de análise.

${ }^{2}$ Endereço para correspondência:

Jean Carlos Natividade. Laboratório de Mensuração. Instituto de Psicologia. Universidade Federal do Rio Grande do Sul. Rua Ramiro Barcelos, 2600, sala 101. CEP 90.035-003. Porto Alegre-RS, Brasil. E-mail: jeannatividade@gmail.com
}

em 1982 que a ciência classificou a doença como síndrome da imunodeficiência adquirida (Mann, Tarantola, \& Netter, 1993). As características das vias de transmissão do vírus da aids (secreções sexuais, sangue e leite materno) ressaltam a influência dos comportamentos das pessoas na disseminação da doença. Em busca de soluções para os problemas advindos com a doença, o mundo envidou esforços na concretização de estratégias para deter o aumento do número de pessoas infectadas por HIV.

A adoção de práticas preventivas, de forma a reduzir as probabilidades de contato com o HIV, apresenta-se como uma dessas estratégias. Ao encontro dela, a investigação dos modelos de previsão de comportamento salienta seus determinantes cognitivos, tais como: conhecimento, crenças, 
normas subjetivas, atitudes (Stroebe \& Stroebe, 1995) e representações sociais (Camargo, Barbará, \& Bertoldo, 2007). Destacam-se no Brasil as ações de práticas preventivas ao contágio por HIV que valorizam o fornecimento de informações científicas à população (Ministério da Saúde, 2008), fundamentadas na ideia de que empossadas das informações as pessoas passem a agir de forma mais segura frente às vias de transmissão do vírus.

O conhecimento científico, produto de especialistas, gera informações capazes de identificar, categorizar, combater e evidenciar as repercussões da aids na vida humana, a partir de uma proposta de neutralidade de valores, sua apropriação pelos leigos proporciona a construção de um conhecimento de senso comum - as representações sociais (Moscovici, 1981) - permeado de valores, crenças, atitudes e normas sociais (Wagner, 1998). Essas representações fornecem elementos de caracterização de como grupos de pessoas pensam e agem diante da aids. Nesse processo, destaca-se o papel da ciência como fornecedora de conhecimento; e das representações sociais como denotativa do conhecimento que as pessoas compartilham sobre a aids.

Segundo Wagner (1998), representação social é, ao mesmo tempo, um conteúdo mental estruturado e conscientemente compartilhado com outros membros do grupo social (que possui aspectos cognitivos, avaliativos, afetivos e simbólicos sobre um fenômeno social relevante e capaz de assumir formas metafóricas ou imagéticas) e um processo público de criação, mudança, elaboração e difusão do conhecimento compartilhado. Jodelet (2001) as define como uma forma de conhecimento elaborada socialmente $\mathrm{e}$ partilhada entre as pessoas, que converge para a construção de uma realidade comum a um grupo social e possui um objetivo prático.

As representações sociais estabelecem relações de simbolização (substituindo o objeto) e de interpretação (conferindo-lhe significações) entre sujeito e objeto. Para Doise (2001), elas embasam e norteiam posicionamentos específicos diante de objetos em relações sociais, e organizam os processos simbólicos que intervêm nessas relações. Abric (2001) defende que as representações explicam a realidade, orientam práticas sociais e justificam tomadas de posição depois que elas foram tomadas, além de definirem a identidade dos grupos.

Em sua construção, por meio da interação entre os membros do grupo, as representações são caracterizadas também por situações extragrupo, como o conhecimento proveniente de especialistas que é repassado pelas fontes de informação, como lembrou Cabecinhas (2004) em seu trabalho de revisão. Como a comunicação, sobretudo a linguagem, configura o sistema formador de representações sociais (Moscovici, 1981), as principais técnicas de descrição delas perpassam pela identificação de símbolos linguísticos compartilhados pelas pessoas. Reconhecer os elementos que constituem essas representações, e suas conexões com o conhecimento científico e com as fontes de informação, fornece pistas sobre as cognições que se relacionam com os comportamentos perante a doença e sobre o nível de vinculação dessas cognições com eventos originados fora do grupo.

Em pesquisa com estudantes mexicanos de ensino médio, Flores-Palacios e Leyva-Flores (2003) identificaram como centrais para a representação social da aids os elementos: morte, doença e sexo. No Brasil, Tura (1998) encontrou como elementos centrais da representação social da aids para adolescentes: morte, sexo, camisinha e doença. A aids estava associada tanto à doença capaz de matar quanto à prevenção sexual. Ao encontro dessa última associação, Camargo (2000), em um grupo de jovens universitários, encontrou como elementos centrais da representação social da aids: sexo e prevenção, o que caracterizou a relação da aids com a noção de prevenção sexual. Thiengo, Oliveira e Rodrigues (2002) chegaram às mesmas conclusões sobre a representação social da aids ao pesquisarem adolescentes.

Além da conclusão de Flores-Palacios e Leyva-Flores (2003) de que a representação social da aids estava vinculada a uma doença mortal causada pelo sexo, os autores apontaram que, embora os participantes considerassem a aids como uma doença suscetível a qualquer um, ela não era vista como comum para seu grupo de pares. Madeira (1998) chegou à inferência semelhante, a partir de uma pesquisa com um grupo de pessoas de nove a 22 anos de idade, em que apontou os elementos morte e prevenção associados à representação social da aids na maior parte das entrevistas realizadas. Esse autor verificou que o grupo temia a aids pela possibilidade da morte, porém, consideravam-se imunes a ela, colocando-a sob a alçada do outro. Enquanto a prevenção, o uso do preservativo, estava relacionada à desconfiança, e servia como um objeto de proteção contra o grupo dos que eram diferentes deles.

A ideia de dissociação da aids de seu grupo de pares também foi apresentada por Vieira, Amaral e Saldanha (2007), em pesquisa com adolescentes, em que identificaram que os participantes do sexo masculino apresentavam representação social da aids marcada por elementos denotativos de um sentimento de invulnerabilidade e distante de sua realidade, enquanto as mulheres associavam o tema à doença, morte e sofrimento decorrentes da desinformação. Joffe e Bettega (2003), em pesquisa sobre representação social da aids de adolescentes zambianos, constataram que as mulheres se implicavam como possíveis vetores de transmissão da doença, enquanto o mesmo não ocorria com os homens.

A variável sexo e sua relação com as representações sociais da aids foi também explorada no estudo de Azevedo, Fonseca, Coutinho e Saldanha (2006), com adolescentes do sexo feminino, em que apontou elementos relacionados ao contágio como: doença, camisinha, prevenir; e elementos de carga afetiva como: preconceito, ruim, descuido, arrependimento. Camargo, Bertoldo e Barbará (2009) constataram, para adolescentes, associação entre ser do sexo masculino e representar a aids com elementos de características pragmáticas, como formas de transmissão e prevenção. Associação 
similar à encontrada por Thiengo, Oliveira e Rodrigues (2005), para grupo de adolescentes portadores e não portadores de HIV, entre os quais, além disso, verificaram relações entre o sexo feminino e elementos configuradores de um sentimento de empatia para com os portadores do vírus HIV.

Em outro estudo realizado com adolescentes, Camargo e cols. (2007) demonstraram que a representação social da aids era formada pelos elementos: morte, doença, sexo, preservativo, tristeza, prevenção, medo e sofrimento. Quando realizaram o teste de confirmação do núcleo central, a partir da marcação dos elementos que os adolescentes consideravam os mais importantes, constataram a centralidade de todos, exceto tristeza. Além desses, foi confirmada a centralidade de elementos que apareceram como situados no sistema periférico: preconceito, irresponsabilidade e descuido.

A respeito do conhecimento científico que as pessoas possuem sobre HIV e aids, encontram-se pesquisas em que se salienta apenas um aspecto do conhecimento da aids, como o conhecimento sobre formas de transmissão e prevenção (Carey, Morrison-Beedy, \& Johnson, 1997; Ferreira, 2003, 2008; Martins e cols., 2006; Trajman e cols., 2003). E outras que pretendem avaliar mais que um fator do conhecimento, como: conceituação HIV e aids, transmissão, infecção pelo vírus e tratamento, formas de prevenção (Camargo, Barbará, $\&$ Bertoldo, 2005; Camargo e cols., 2007, Lazzarotto e cols., 2009).

Ferreira (2003), em pesquisa com dados do ano de 1998 da população brasileira em geral, concluiu que o nível de conhecimento sobre formas de transmissão do HIV aumentou conforme ocorreu melhorias nas condições socioeconômicas, sendo as maiores diferenças entre as classes A e E. Ao encontro desses resultados, a pesquisa de Trajman e cols. (2003), com adolescentes de escolas públicas e privadas, constatou que a baixa renda familiar era preditora de baixo nível de conhecimento sobre transmissão e prevenção do HIV.

Em pesquisa para construção e validação de um teste de conhecimento para as formas de transmissão e prevenção do HIV, Carey e cols. (1997), nos Estados Unidos, verificaram que o nível de conhecimento sobre esse assunto aumentava conforme o acréscimo do grau de escolaridade. Martins e cols. (2006), ao pesquisarem estudantes do ensino médio de escolas públicas e privadas, também concluíram que ter maior grau de escolaridade era preditor de maior nível de conhecimento sobre prevenção às doenças sexualmente transmissíveis e à aids, além de outros preditores como: ser mulher, ser branco, ser solteiro e estudar em escola privada.

Ferreira (2003) destacou que as mulheres mostraram-se mais bem informadas que os homens em relação à transmissão do HIV, inclusive entre os jovens de 16 a 24 anos e entre diferentes classes socioeconômicas. Posteriormente, Ferreira (2008) comparou dados dos anos de 1998 e 2005 da população brasileira sobre o conhecimento da transmissão do HIV e notou acréscimos em seu nível para os homens, de forma a não mais caracterizar diferenças entre esses e as mulheres.
Lazzarotto e cols. (2009) verificaram uma alta frequência de respostas erradas sobre o conhecimento de conceitos, da transmissão e da prevenção do HIV e aids, em uma amostra de participantes da terceira idade. Resultados compatíveis com os achados de Camargo e cols. (2007), para a população adolescente, em que demonstrou que o nível de conhecimento dos estudantes do ensino médio sobre o HIV e sua transmissão, a infecção pelo vírus e tratamento, e as formas de prevenção do HIV era insuficiente para considerálos cientificamente bem informados a respeito. Esses dados corroboram os estudos de Camargo e cols. (2005) que salientaram um baixo nível de conhecimento sobre HIV e aids entre estudantes do ensino médio e Camargo e Botelho (2007) que constataram falhas no conhecimento sobre transmissão e prevenção ao HIV para a mesma população.

Martins, Nunes, Muñoz-Silva e Sánchez-García (2008), em um estudo com estudantes universitários de Portugal e Espanha, identificaram como principais fontes de informação sobre aids: revistas/livros, professores e televisão/ cinema; e concluíram que quanto mais jovens eram os estudantes, maior era a frequência do uso da fonte professores. Resultados semelhantes aos encontrados por Camargo e Botelho (2007), com amostra de estudantes brasileiros do ensino médio, que apontaram como principais fontes de informação sobre o assunto: a escola e a televisão, além de revistas, amigos e jornais que também se destacaram, porém como fontes secundárias. Além disso, esses autores detectaram que a qualidade do conhecimento sobre a aids estava relacionada à fonte de informação. Os estudantes que declararam ter os amigos como principal fonte, obtiveram baixo nível de conhecimento sobre a transmissão do HIV.

Camargo e cols. (2007) ressaltaram que o nível de conhecimento científico que os estudantes possuíam sobre o HIV e aids provocava diferenças nas suas representações sociais da doença. A representação da aids dos alunos com menor nível de conhecimento apresentou mais frequentemente o elemento tristeza, ligado à dimensão afetiva da representação. Já os alunos com maior nível de conhecimento evocaram mais frequentemente o elemento sangue, ligado ao conhecimento biomédico (científico), sobre a transmissão do vírus.

Neste estudo, pretendeu-se verificar as relações entre os valores simbólicos atribuídos aos elementos descritores da representação social da aids, o nível de conhecimento científico sobre o HIV e aids, e as fontes de obtenção de informações sobre aids para estudantes do ensino médio de uma escola pública. Identificar o valor simbólico dos elementos significa constatar o grau de importância que os participantes conferem aos elementos como representativos do objeto em estudo. Embasado nas pesquisas com essa população (Camargo e cols., 2007), selecionaram-se para a verificação da atribuição de valor simbólico os elementos: morte, sexo, descuido, doença, prevenção, medo, sofrimento, preconceito, responsabilidade, preservativo e tristeza. 


\section{Método}

\section{Participantes}

Participaram da pesquisa 150 estudantes do ensino médio de uma escola pública da região sul do Brasil, com idades entre 14 e 25 anos ( $M=16$ anos, $D P=1,40$ anos). Dentre todos, $56 \%$ eram do sexo feminino; $59 \%$ estudavam no período noturno e $41 \%$ no período vespertino; $53 \%$ estavam no primeiro ano do ensino médio, $23 \%$ no segundo ano e $21 \%$ no terceiro ano. Quanto à experiência sexual, $58 \%$ deles declararam ter realizado sexo alguma vez na vida (seja oral, vaginal ou anal).

\section{Instrumentos}

Utilizou-se como instrumento de pesquisa um questionário autoaplicável de respostas fechadas, com quatro blocos de questionamentos: questões sociodemográficas; questão sobre representações sociais da aids; questão sobre fontes de informação sobre aids; questão sobre o conhecimento científico a respeito de HIV e aids. As questões sociodemográficas perguntavam sobre o sexo, a idade, experiência sexual, o turno e a série em que estudavam os adolescentes.

A questão sobre as representações sociais da aids apresentava os elementos "tristeza, descuido, sexo, morte, doença, prevenção, medo, sofrimento, preconceito, responsabilidade e preservativo" em itens seguidos de uma escala numérica variável entre quatro pontos, em que os estudantes deveriam julgar o quanto lhes era possível pensar em aids sem considerar o elemento em questão, tal que um (1) correspondia a "certamente não é possível" e quatro (4) correspondia a "certamente é possível".

A questão sobre as fontes de informação dispunha-se em oito itens escalares, variáveis de um (1) a cinco (5), para que os participantes avaliassem a quantidade de informação recebida, em que um (1) correspondia a pouquíssimas informações e cinco (5) correspondia a muitíssimas informações sobre cada uma das fontes: internet; amigos; folders e panfletos informativos; pais e/ou parentes; escola e/ou professores; televisão e/ou rádio; médicos e/ou profissionais de saúde; revistas e/ou jornais.

No bloco referente ao conhecimento científico sobre HIV e aids, apresentava-se o teste de conhecimento de Camargo e cols. (2005), composto de 24 itens divididos em três subtestes, no formato de resposta objetiva (verdadeiro, falso, ou não sei). Cada subteste abordava uma de três dimensões do conhecimento científico sobre aids: (a) subteste 1: HIV e sua transmissão (10 itens); (b) subteste 2: infecção e tratamento (6 itens); (c) subteste 3: prevenção (8 itens). Para o participante ser considerado bem informado cientificamente, o teste exigia um número mínimo de acertos em cada um dos subtestes: sete questões no $1^{\circ}$, três no $2^{\circ}$ e cinco no $3^{\circ}$.

\section{Procedimento}

\section{Coleta de dados}

Durante as aplicações do questionário, nas salas de aula do colégio e nos horários de aulas, forneceram-se informações aos participantes sobre a voluntariedade e anonimato da participação, bem como do objetivo do trabalho e do sigilo em relação às respostas prestadas. Também lhes foi informado sobre a forma de responder os itens, destacando o fato de serem questionários respondidos individualmente. Assim que terminavam de responder, os questionários eram colocados em uma urna fechada e os estudantes permaneciam em seus lugares até que todos respondessem.

\section{Análise dos dados}

Os questionários foram analisados em conjunto por meio de cálculos descritivos e inferenciais, esses últimos com nível de significância de $p \leq 0,05$. As variáveis sociodemográficas tiveram suas frequências calculadas e também serviram como variáveis independentes nas outras análises.

Os dados provenientes dos itens do questionamento dos elementos da representação social e das fontes de informação sobre aids tiveram suas médias $(M)$ e desvios-padrão $(D P)$ calculados com intuito de descrever o valor simbólico dos elementos e o grau de obtenção de informação de cada uma das fontes. Para analisar o nível de valor simbólico de cada elemento, estipulou-se que quanto menos possível era pensar em aids sem considerar o elemento em questão, maior o valor simbólico do elemento. Assim, inverteram-se os valores das respostas dos itens de questionamento dos elementos da representação social. As possíveis diferenças entre as médias, tanto dos elementos da representação quanto das fontes de informação, foram testadas através da ANOVA de um fator para medidas repetidas. Com a finalidade de testar as ligações entre os elementos e formar agrupamentos entre os mais relacionados elaborou-se uma matriz de correlações entre eles e efetuou-se uma análise fatorial.

Calcularam-se os escores dos estudantes no teste de conhecimento para cada subteste através da soma das respostas corretas, de forma que a cada resposta correta atribuía-se um (1) ponto; às respostas erradas, àquelas que eles assinalavam que não sabiam responder e às em branco atribuía-se zero (0) ponto. Também se caracterizou o grau de conhecimento científico sobre HIV e aids por meio de um escore geral (de 0 a 24 pontos) a partir de todas as respostas corretas ao teste de conhecimento. Optou-se por essa análise a fim de se quantificar o nível de conhecimento geral sobre HIV e aids. Para testar possíveis diferenças entre os participantes com escores diferentes no teste, dividiu-se a amostra em dois grupos através da mediana do escore geral do teste de conhecimento. 
As relações entre os elementos da representação social, as fontes de informação e o conhecimento científico foram verificadas através de correlações de Pearson, e teste t de Student para amostras independentes entre os grupos formados a partir da divisão dos escores no teste de conhecimento.

\section{Considerações éticas}

Após obtenção de aprovação do Comitê de Ética em Pesquisa com Seres Humanos da Universidade Federal de Santa Catarina (Protocolo n ${ }^{\circ}$ 200/09), contatou-se a escola, solicitou-se autorização para os pais dos menores de 18 anos de idade e agendaram-se os momentos de coleta de dados com todos aqueles que consentiram participar.

\section{Resultados}

As médias de idades dos estudantes entre as três séries mostraram-se diferentes, os estudantes do primeiro ano tinham em média 15,29 anos ( $D P=0,71$ anos); os do segundo ano, média de 16,68 anos $(D P=0,91$ anos); e os do terceiro ano, média de 17,53 anos $(D P=1,68)[F(2)=60,40 ; p<$ $0,001]$. As idades dos estudantes do período vespertino $(M=$ $15,11 ; D P=0,58)$ e noturno $(M=16,78 ; D P=1,39)$ também foram diferentes $[t(123,03)=9,99 ; p<0,001]$. A experiência sexual estava associada: à série em que se encontravam os estudantes $\left[X^{2}(2, N=149)=15,81 ; p<0,001\right]$, de maneira que $43 \%$ dos estudantes do primeiro ano haviam tido experiência sexual, $76 \%$ dos estudantes do segundo ano e $75 \%$ dos estudantes do terceiro ano; ao turno que estudavam $\left[X^{2}(1, N\right.$ $=149)=27,93 ; p<0,001]$ de forma que $32 \%$ dos estudantes do período vespertino já haviam tido experiência sexual, enquanto $75 \%$ daqueles do período noturno declaram já ter feito sexo; e ao sexo $\left[X^{2}(1, N=149)=6,26 ; p<0,05\right]$ sendo que $69 \%$ dos homens já haviam tido experiência sexual, contra $49 \%$ das mulheres.

Os itens sobre o valor simbólico dos elementos variavam de 1 a 4 pontos. Quanto mais próximo do 4, maior valor simbólico possuía o elemento; a proximidade do 1 indicava menor valor simbólico do elemento em relação ao objeto em questão (a aids). Cada elemento obteve um valor de média e desvio-padrão, conforme a Tabela 1.
Tabela 1

Médias e desvios-padrão da valorização simbólica dos elementos

\begin{tabular}{lcc}
\hline \multicolumn{1}{c}{ Elementos } & $\boldsymbol{M}$ & $\boldsymbol{D P}$ \\
\hline Doença & 3,46 & 0,94 \\
Medo & 3,36 & 0,93 \\
Preservativo & 3,36 & 1,04 \\
Descuido & 3,32 & 0,97 \\
Prevenção & 3,30 & 0,99 \\
Sofrimento & 3,30 & 1,04 \\
Responsabilidade & 3,27 & 1,03 \\
Tristeza & 3,27 & 0,94 \\
Preconceito & 3,04 & 1,10 \\
Morte & 3,03 & 1,16 \\
Sexo & 2,84 & 1,24 \\
\hline
\end{tabular}

Os elementos que apresentaram maiores médias foram doença e medo, entretanto, esses só se mostraram com médias significativamente maiores que a dos elementos preconceito, morte e sexo. Os elementos preservativo, descuido, prevenção, sofrimento, responsabilidade e tristeza também tiveram suas médias significativamente maiores que a do elemento $\operatorname{sexo}[F(7,91)=6,99 ; p<0,001]$. Entre homens e mulheres o elemento preservativo alcançou média maior entre essas $(M$ $=3,52 ; D P=0,88)$ que entre aqueles $(M=3,15 ; D P=1,19)$ $[t(116,31)=2,12 ; p<0,05]$. O turno que os participantes estudavam apontou diferenças entre médias para o elemento preconceito, tal que para os estudantes do período noturno $(M=3,19 ; D P=1,02)$ o elemento revelou médias maiores que para os estudantes do período vespertino $(M=2,82 ; D P$ $=1,18)[t(117,5)=2,00 ; p<0,05]$. Em relação às diferenças por escolaridade, o elemento sexo apresentou-se com maior média para os estudantes do terceiro ano $[F(2)=5,99 ; p<$ $0,01]$. A experiência sexual não caracterizou diferença para média de nenhum elemento.

As relações entre os elementos da representação social evidenciaram um número considerável de correlações entre eles, como expõe a matriz de correlações na Tabela 2.

Tabela 2

Coeficientes de correlação de Pearson entre os elementos da representação social da aids

\begin{tabular}{|c|c|c|c|c|c|c|c|c|c|c|}
\hline & 1 & 2 & 3 & 4 & 5 & 6 & 7 & 8 & 9 & 10 \\
\hline (1) Tristeza & - & & & & & & & & & \\
\hline (2) Descuido & $0,34^{* *}$ & - & & & & & & & & \\
\hline (3) Sexo & $0,25^{* *}$ & $0,21 *$ & - & & & & & & & \\
\hline (4) Morte & $0,38 * *$ & $0,29 * *$ & $0,46^{* *}$ & - & & & & & & \\
\hline
\end{tabular}


Tabela 2

(continuação)

\begin{tabular}{|c|c|c|c|c|c|c|c|c|c|c|}
\hline & 1 & 2 & 3 & 4 & 5 & 6 & 7 & 8 & 9 & 10 \\
\hline (5) Doença & $0,44 * *$ & $0,38 * *$ & $0,37 * *$ & $0,51 * *$ & - & & & & & \\
\hline (6) Prevenção & $0,30 * *$ & $0,24 * *$ & $0,20 *$ & $0,24 * *$ & $0,35 * *$ & - & & & & \\
\hline (7) Medo & $0,31 * *$ & $0,37 * *$ & $0,18 *$ & $0,50 * *$ & $0,51 * *$ & $0,43 * *$ & - & & & \\
\hline (8) Sofrimento & $0,51 * *$ & $0,40 * *$ & 0,13 & $0,46^{* *}$ & $0,45 * *$ & $0,34 * *$ & $0,57 * *$ & - & & \\
\hline (9) Preconceito & $0,29 * *$ & 0,30 & $0,28 * *$ & $0,33 * *$ & $0,27 * *$ & $0,20 *$ & $0,40 * *$ & $0,56 * *$ & - & \\
\hline (10) Responsabilidade & $0,26 * *$ & $0,40 * *$ & $0,24 * *$ & $0,25 * *$ & $0,36 * *$ & $0,36^{* *}$ & $0,46^{* *}$ & $0,42 * *$ & $0,23 * *$ & - \\
\hline (11) Preservativo & $0,33 * *$ & $0,30 * *$ & $0,21 * *$ & $0,22 * *$ & $0,42 * *$ & $0,43 * *$ & $0,39 * *$ & $0,34 * *$ & $0,22 * *$ & $0,50 * *$ \\
\hline
\end{tabular}

$* p<0,05 * * p<0,01$

Um número alto de correlações significativas entre os elementos era esperado, tendo em vista que se referem ao mesmo objeto de representação. Para averiguar a existência de relações fortes o suficiente para agrupar os elementos, efetuou-se uma análise fatorial. A extração dos componentes principais indicou que a matriz era fatorizável $(K M O=$ 0,81 ). Quanto ao número de fatores, obtiveram-se os seguintes dados: eigenvalue maior que 1 - três fatores; Scree plot de Catell - três fatores; variância explicada para três fatores $-60,58 \%$. Ao analisar as matrizes de componentes com três fatores (rotação varimax), verificou-se, entre os elementos com cargas acima de 0,30 , que tristeza mostrou peso semelhante entre todos os fatores; doença apresentou carga entre os fatores 1 e 3 ; descuido entre os fatores 1 e 3 ; morte entre 2 e 3 ; medo entre 1 e 2 ; sofrimento entre 1 e 2 . A Tabela 3 permite uma visualização do agrupamento dos elementos em fatores, demonstram-se aqueles com cargas acima de 0,55.

Tabela 3

Matriz fatorial dos elementos da representação social da aids

\begin{tabular}{lccc}
\hline & Fator 1 & Fator 2 & Fator 3 \\
\hline Responsabilidade & 0,73 & - & - \\
Preservativo & 0,74 & - & - \\
Prevenção & 0,65 & - & - \\
Descuido & 0,57 & - & - \\
Preconceito & - & 0,87 & - \\
Sofrimento & - & 0,74 & - \\
Medo & - & 0,57 & - \\
Sexo & - & - & 0,82 \\
Morte & - & - & 0,72 \\
Doença & - & - & 0,59 \\
\hline
\end{tabular}

O fator 1 apontou os elementos responsabilidade e preservativo como os de maiores cargas, acompanhados de prevenção e descuido. O fator 2 trouxe o elemento preconceito com alto valor de carga, associado aos elementos sofrimento e medo. Já o fator 3 destacou o elemento sexo como de maior carga, seguido dos elementos morte e doença.

O grau de obtenção de informações a partir das fontes de informação foi caracterizado por meio da média e desviopadrão para cada fonte. Os itens variavam de 1 a 5 pontos, quanto mais próximo do 5 , mais informações recebiam da fonte; quanto mais próximo do 1 , menos informações recebiam. A Tabela 4 descreve os valores das médias e desviospadrão para cada fonte.

Tabela 4

Médias e desvios-padrão da obtenção de informação das fontes

\begin{tabular}{lcc}
\hline \multicolumn{1}{c}{ Fontes de informação } & $\boldsymbol{M}$ & $\boldsymbol{D P}$ \\
\hline Escola e/ou professores & 3,74 & 1,18 \\
Médicos e/ou profissionais de saúde & 3,74 & 1,31 \\
Pais e/ou parentes & 3,62 & 1,46 \\
Televisão e/ou rádio & 3,35 & 1,23 \\
Folders e/ou panfletos informativos & 3,30 & 1,36 \\
Internet & 3,12 & 1,40 \\
Amigos & 2,83 & 1,32 \\
Revistas e/ou jornais & 2,80 & 1,17 \\
\hline
\end{tabular}

Os participantes, em geral, declararam receber mais informações da escola e/ou professores, de médicos e/ou profissionais de saúde e de pais e/ou parentes do que das outras fontes $[F(6,02)=14,40 ; p<0,001]$, sem diferenças entre as 
médias nas três fontes com maiores médias. As fontes que obtiveram menor média foram revistas e/ou jornais e amigos, e foram significativamente menores que as médias das três fontes com médias mais elevadas. Em relação ao turno em que estudavam, a fonte pais e/ou parentes mostrou média maior para os do turno vespertino $(M=3,92 ; D P=1,20)$ que para os do turno noturno $(M=3,39 ; D P=1,54)[t(141,84)=$ 2,30; $p<0,05]$; a fonte revistas e/ou jornais acusou média de obtenção de informação maior para os alunos do turno noturno $(M=3,01 ; D P=1,23)$ comparada aos do turno vespertino $(M=2,48 ; D P=1,09)[t(138,50)=2,80 ; p<0,001]$. Quanto às diferenças entre a escolaridade dos participantes, a fonte escola e/ou professores demonstrou-se com média maior para os alunos do segundo ano, em relação aos do primeiro $[F(2)=3,89 ; p<0,05]$; a fonte revistas e/ou jornais revelou média mais elevada entre os alunos do terceiro ano que entre os do primeiro $[F(2)=4,47 ; p=0,01]$. Sexo e experiência sexual não discriminaram obtenção de informações para nenhuma das fontes.

Quatro fontes de informação correlacionaram-se com sete elementos da representação social, como apresenta a Tabela 5.

Tabela 5

Coeficientes de correlação de Pearson significativos entre as fontes de informação e os elementos da representação social da aids

\begin{tabular}{lcccc}
\hline & Internet & Amigos & $\begin{array}{c}\text { Pais e/ou } \\
\text { parentes }\end{array}$ & $\begin{array}{c}\text { Televisão } \\
\text { e/ou rádio }\end{array}$ \\
\hline Tristeza & - & - & - & $0,17^{*}$ \\
Sexo & - & $0,19^{*}$ & - & - \\
Prevenção & $-0,20^{*}$ & - & - & - \\
Medo & $-0,27^{* *}$ & - & - & - \\
Sofrimento & $-0,19^{*}$ & - & - & - \\
Responsabilidade & - & $0,18^{*}$ & - & - \\
Preservativo & - & - & $0,18^{*}$ & - \\
\hline
\end{tabular}

$* p<0,05 * * p<0,01$

A fonte internet destacou-se por correlacionar-se negativamente com três elementos da representação: prevenção, medo e sofrimento. De forma que o incremento da obtenção de informação dessa fonte diminuiu o valor simbólico desses elementos. A fonte amigos correlacionou-se positivamente com a valorização simbólica dos elementos sexo e responsabilidade, enquanto pais e/ou parentes com a do elemento preservativo, e televisão e/ou rádio com a do elemento tristeza, de modo que quanto mais informações receberam dessas fontes, mais os participantes atribuíram valor simbólico ao respectivo elemento correlacionado.

O escore geral no teste de conhecimento, variável de 0 a 24 pontos, revelou valores entre 0 a 14 pontos na amostra estudada. A média de acertos foi de 6,20 pontos $(D P=3,00$ pontos, $M d=6,00$ pontos $)$. Os homens ( $M=7,17 ; D P=3,13$ pontos) obtiveram maiores escores no teste que as mulheres $(M=5,43 ; D P=2,67$ pontos $)[t(148)=3,60 ; p<0,001]$. As diferenças entre os escores no teste de conhecimento dos estudantes do período vespertino e noturno, dos que já haviam e não haviam tido experiência sexual, bem como as variações dos escores entre os alunos de séries diferentes não foram significativas. Nenhum estudante atingiu a pontuação mínima necessária para ser considerado bem informado em cada um dos subtestes.

A valorização simbólica dos elementos da representação social e a obtenção de informações das fontes de informação não apresentaram médias diferentes entre os estudantes com escores gerais, e nos subtestes, abaixo e acima da mediana no teste de conhecimento. O grau de conhecimento científico também não se correlacionou com os elementos da representação e com as fontes de informação.

\section{Discussão}

A representação social da aids denotou os elementos doença e medo como os considerados com maior valor simbólico para essa população. $\mathrm{O}$ alto valor simbólico do elemento doença, um descritor da aids, também se mostrou presente em uma série de estudos para populações com idades e escolaridades similares (Azevedo e cols., 2006; Camargo e cols., 2007; Flores-Palacios \& Leyva-Flores, 2003; Madeira, 1998; Tura, 1998). O elemento medo correlacionou-se com o elemento morte, de forma semelhante aos achados de Madeira (1998) que relacionou o medo da aids a sua possibilidade de ocasionar a morte.

O elemento sexo, com média menor que a maioria dos elementos (doença, medo, preservativo, descuido, prevenção, sofrimento, responsabilidade e tristeza), demonstrou-se menos representativo da aids do que esses. Isso caracterizou uma desvalorização desse elemento, se comparado aos estudos de Camargo (2000), Camargo e cols. (2007), Flores-Palacios e Leyva-Flores (2003), Thiengo e cols. (2002) e Tura (1998). Tal resultado, unido às médias altas dos elementos preservativo e prevenção, bem como suas correlações com o elemento sexo, coaduna a ideia de que o sexo em si não estaria relacionado à representação da aids; mas sim, o sexo sem proteção estaria vinculado à doença. Ademais, o elemento sexo foi mais valorizado pelos estudantes do terceiro ano, que eram mais velhos e já haviam tido alguma experiência sexual, o que aproxima esse elemento da realidade experimentada por esse grupo.

A valorização do elemento preservativo foi maior entre as mulheres que entre os homens, e isso é compatível com os dos dados de Camargo (2000) que identificou que para as mulheres a aids estaria ligada ao sexo e à morte, devendo ser prevenida por meio da adoção do uso de preservativo. Enquanto para os homens a aids aparecia como uma doença temível que deveria ser prevenida, entretanto não mencionavam o uso do preservativo. Esse resultado também se assemelha as conclusões de Joffe e Bettega (2003) e Vieira e cols. (2007) de 
sentimento de invulnerabilidade ao HIV dos homens e a consequente não necessidade de utilização do preservativo. Esses dados, associados ao fato dos homens terem apresentado maior experiência sexual que as mulheres, apontam para a hipótese: o elemento preservativo teria maior valor simbólico apoiado em noções abstratas (desconectadas da realidade experimentada) que em situações concretas. A confirmação dessa hipótese revelaria dados importantes para o controle da epidemia, pois destacaria uma representação de um objeto de prevenção dissociado da prática preventiva.

O preconceito foi considerado mais representativo da aids para os estudantes do período noturno comparado aos do período vespertino. A idade mais elevada dos estudantes do período noturno, a maior frequência de alunos com experiência sexual nesse período e a correlação com o elemento preservativo destacam o preconceito como um elemento mais valorizado pelos estudantes que vivenciavam situações concretas de possibilidade de contágio pela aids. Além disso, as significativas correlações com os elementos sofrimento e medo salientam uma característica afetiva do elemento para o grupo.

$\mathrm{O}$ agrupamento em fatores entre os diversos elementos da representação social da aids mostrou coerência interpretativa possível de ser aventada em três únicos fatores: (1) responsabilidade diante do contágio; (2) medo das consequências do contágio; (3) descrição da aids.

$\mathrm{O}$ fator 1 agrupou elementos relacionados à responsabilidade pessoal perante o contato com a aids (responsabilidade, preservativo, prevenção, descuido). Esse fator destacou-se como de característica prescritiva de comportamento diante do objeto em estudo, agrupando elementos associados a orientações de práticas, corroborando a proposta de Abric (2001) e Doise (2001) para as representações sociais. Ressalta-se que o comportamento de prevenção, o uso de preservativo, estaria ligado à noção de responsabilidade em usá-lo e de seu opositor ao não usá-lo: o descuido; noção também identificada por Camargo e cols. (2009), FloresPalacios e Leyva-Flores (2003) e Thiengo e cols. (2002) e como representativa da aids para adolescentes.

O fator 2 agrupou elementos simbólicos de características afetivas e emocionais (preconceito, sofrimento e medo) e salientou um aspecto afetivo da representação social da aids para esse grupo, tal como teorizou Wagner (1998) sobre as representações sociais, de modo que a aids associava-se ao medo, que por sua vez estaria relacionado à possibilidade de sofrimento e ao preconceito. Elementos de carga afetiva também já foram encontrados em pesquisas sobre representação social da aids para adolescentes e estavam associados às mulheres (Azevedo e cols., 2006; Thiengo e cols. 2005).

O fator 3 agrupou elementos que descreveriam e explicariam a aids (sexo, morte e doença). De forma a indicar que o grupo identificou a aids como uma doença que levaria a morte e poderia ser causada pelo sexo, como destacaram $\mathrm{Ca}$ margo e cols. (2007), Flores-Palacios e Leyva-Flores (2003) e Tura (1998), em pesquisas com populações de idade e escolaridade semelhantes.
Com relação ao teste de conhecimento sobre HIV e aids, nenhum estudante obteve a pontuação mínima necessária para ser considerado bem informado nos subtestes. Resultados de baixos níveis, ou de falhas, de conhecimento científico sobre aids para essa população já foram observados por Camargo e cols. (2005), Camargo e Botelho (2007) e Camargo e cols. (2007), e em população com mais idade por Ferreira (2003, 2008) e Lazzarotto e cols. (2009). Um maior nível de conhecimento entre os homens acompanhou os dados de Ferreira (2008) que, embora não tenha verificado diferenças entre os sexos, constatou um acréscimo no nível de conhecimento entre homens do ano 1998 a 2005.

O conhecimento científico sobre HIV e aids não se relacionou com as variáveis sociodemográficas, inclusive não houve diferenças entre os estudantes com graus de escolaridade diferentes, resultado discordante dos achados de Carey e cols. (1997) e Martins e cols. (2006). Também não houve relação entre os níveis de conhecimento e a obtenção de informação das fontes, e níveis de conhecimento e a valorização simbólica dos elementos da representação social.

Considerando que as representações sociais também são originadas pelo conhecimento gerado no universo reificado - o conhecimento científico (Moscovici, 1981) -, é provável que essa inexistência de relações seja consequência da pouca diferenciação entre os participantes com mais e menos conhecimento científico, o que atenta para a proposta de construção de um instrumento de medida do conhecimento científico com maior poder discriminativo para novos estudos. Sobretudo para grupos com características semelhantes, como no caso de participantes adolescentes de uma mesma escola, verificar relações entre níveis de conhecimento científico e representações sociais poderá fornecer dados sobre o impacto do conhecimento científico na formação de representações sociais sobre a aids.

De maneira geral, os estudantes declararam ter recebido mais informações sobre aids da escola e/ou professores, de médicos e/ou profissionais de saúde e de pais e/ou parentes, confirmando os dados de Camargo e Botelho (2007). Quanto à obtenção de informação de revistas e/ou jornais e de amigos, neste estudo os estudantes declararam ter recebido menos informações dessas do que das outras fontes questionadas, resultado contrário aos achados de Camargo e Botelho (2007) e de Martins e cols. (2008). As duas principais vias de obtenção de informação declaradas nesse estudo são fontes que estariam capacitadas para assegurar dados científicos sobre a aids, entretanto os resultados no teste de conhecimento indicam que o nível de conhecimento dos estudantes é insatisfatório para serem considerados bem informados sobre aids. Esses dados fornecem subsídios para elaboração de, pelo menos, duas hipóteses: (1) as fontes supostamente capacitadas não fornecem suficientemente o conhecimento científico sobre o assunto; (2) essas vias de informação não conseguem produzir resultados de retenção de informações nos estudantes.

No tocante às fontes de informação e os elementos da representação social, as correlações significativas destacam 
a importância da comunicação extragrupo na constituição das representações sociais, como ressaltou Jodelet (2001) e lembrou Cabecinhas (2004) sobre os meios de comunicação em massa, visto que cada uma das fontes de informação sobre a aids realça singulares símbolos linguísticos ao comunicar o assunto. Os estudantes associaram mais os elementos responsabilidade e sexo à aids, conforme receberam mais informações dos amigos; valorizaram mais o elemento preservativo à medida que obtinham mais informações dos pais e/ou parentes; e quanto mais informações recebiam da televisão e/ou rádio, maior valor simbólico atribuíam ao elemento tristeza. Receber mais informações da internet contribuiu para os estudantes atribuírem menor valor simbólico aos elementos prevenção, medo e sofrimento.

\section{Considerações finais}

O estudo permitiu identificar o nível de valorização atribuída aos elementos da representação social da aids (valorização simbólica), entre estudantes do ensino médio. Considera-se que tal valorização revela o grau de importância atribuída ao elemento como representativo do objeto em questão. Observou-se, por exemplo, que os elementos doença, medo e preservativo foram os mais valorizados pelos respondentes, enquanto o elemento sexo obteve o menor grau de valorização.

Por meio desse sistema de inquérito (importância atribuída ao elemento) foi possível testar as relações entre os elementos, de sorte que indicou três fatores como caracterizadores da representação social da aids: responsabilidade diante do contágio; medo das consequências do contágio; descrição da aids.

Então se testaram as relações entre os elementos e o grau de obtenção de informação das fontes e o nível de conhecimento científico, sendo que para os primeiros as correlações salientaram a importância da comunicação extragrupo na constituição das representações sociais. Já com o conhecimento científico as correlações não se mostraram significativas.

Aliás, nenhum dos testes realizados com o conhecimento científico sobre aids mostrou-se significativo, o que revelou uma importante limitação do estudo. Ainda assim, foi possível constatar um baixo nível desse conhecimento entre os participantes. Dado preocupante, tendo em vista que as fontes de informações sobre o tema apontadas como as mais recorridas deveriam estar capacitadas para fornecer informações sobre o assunto (escola e/ou professores; médicos e/ou profissionais de saúde). Sugere-se, para um próximo estudo, a construção e utilização de um instrumento de medida do conhecimento científico da aids com maior poder discriminativo.

\section{Referências}

Abric, J. C. (2001). O estudo experimental das representações sociais. In D. Jodelet (Org.), As representações sociais (pp. 155-171). Rio de Janeiro: Ed. UERJ.
Azevedo, R. L. W., Fonseca, A. A., Coutinho, M. P. L., \& Saldanha, A. A. W. (2006). Representações sociais da adolescente feminina acerca da sexualidade em tempo de aids. Jornal Brasileiro de Doenças Sexualmente Transmissíveis, 18(3), 204-210.

Cabecinhas, R. (2004). Representações sociais, relações intergrupais e cognição social. Paidéia (Ribeirão Preto), 14(28), 125-137.

Camargo, B. V. (2000). Sexualidade e representações sociais da aids. Revista de Ciências Humanas (Edições Temáticas), (3), 97-110.

Camargo, B. V., Barbará, A., \& Bertoldo, R. B. (2005). Um instrumento de medida da dimensão informativa da representação social da aids. Jornada Internacional, 4; Conferência Brasileira Sobre Representações Sociais: Teoria e Abordagens Metodológicas, 2, 1385-1395.

Camargo, B. V., Barbará, A., \& Bertoldo, R. B. (2007). Concepção pragmática e científica dos adolescentes sobre a aids. Psicologia em Estudo, 12(2), 277-284.

Camargo, B. V., Bertoldo, R. B., \& Barbará, A. (2009). Representações sociais da aids e alteridade. Estudos e Pesquisas em Psicologia, 9(3), 710-723.

Camargo, B. V., \& Botelho, L. J. (2007). Aids, sexualidade e atitudes sobre a proteção contra o HIV. Revista de Saúde Pública, 41(1), 61-68.

Carey, M. P., Morrison-Beedy, D., \& Johnson, B. T. (1997). The HIV-knowledge questionnaire: Development and evaluation of a reliable, valid, and practical selfadministered questionnaire. AIDS and Behavior, 1(1), 61-74.

Doise, W. (2001). Cognições e representações sociais: A abordagem genética. In D. Jodelet (Org.), As representações sociais (pp. 301-320). Rio de Janeiro: Ed. UERJ.

Ferreira, M. P. (2003). Conhecimento e percepção de risco sobre o HIV/aids: Um perfil da população brasileira no ano de 1998. Cadernos de Saúde Pública, 19(Suppl. 2), 213-222.

Ferreira, M. P. (2008). Nível de conhecimento e percepção de risco da população brasileira sobre o HIV/aids, 1998 e 2005. Revista de Saúde Pública, 42(Suppl. 1), 65-71.

Flores-Palacios,F., \&Leyva-Flores, R.(2003). Representación social del SIDA en estudiantes de la Ciudad de México. Salud Publica de México, 45(Suppl. 5), 624-631.

Jodelet, D. (2001). Representações sociais: Um domínio em expansão. In D. Jodelet (Org.), As representações sociais (pp. 17-29). Rio de Janeiro: Ed. UERJ.

Joffe, H., \& Bettega, N. (2003). Social representation of AIDS among Zambiam adolescents. Journal of Health Psychology, 8(5), 616-631.

Lazzarotto, A. R., Kramer, A. S., Hädrich, M., Tonin, M., Caputo, P., \& Sprinz, E. (2009). O conhecimento de HIV/ aids na terceira idade: Estudo epidemiológico no Vale do Sinos, Rio Grande do Sul, Brasil. Ciência e Saúde Coletiva, 13(6), 1833-1840. 
Madeira, M. C. (1998). Aconfiança afrontada: Representações sociais da aids para jovens. In D. Jodelet \& M. Madeira (Orgs.), Aids e representações sociais: À busca de sentidos (pp. 17-46). Natal: Ed. UFRN.

Mann, J., Tarantola, D. J. M., \& Netter, T. W. (1993). A aids no mundo. Rio de Janeiro: ABIA/IMS-UERJ/Relume Dumará.

Martins, L. B. M., Costa-Paiva, L. H. S., Osis, M. J. D., Sousa, M. H., Pinto-Neto, A. M., \& Tadini, V. (2006). Fatores associados ao uso de preservativo masculino e ao conhecimento sobre DST/aids em adolescentes de escolas públicas e privadas do Município de São Paulo, Brasil. Cadernos de Saúde Pública, 22(2), 315-323.

Martins, A. T., Nunes, C, Muñoz-Silva, A., \& SánchezGarcía, M. (2008). Fontes de informação, conhecimentos e uso do preservativo em estudantes universitários do Algarve e de Huelva. Psico, 39(1), 7-13.

Ministério da Saúde. (2008). Resposta positiva: A experiência do Programa Brasileiro de DST e aids. Brasília, DF: PNDST/AIDS.

Moscovici, S. (1981). On social representation. In J. P. Forgas (Ed.), Social cognition (pp. 181-209). London: Academic Press.

Stroebe, W., \& Stroebe, M. S. (1995). Psicologia social e saúde. Lisboa: Instituto Piaget.

Thiengo, M. A., Oliveira, D. C., \& Rodrigues, B. M. R. D. (2002). Adolescentes, aids e práticas de proteção: Uma abordagem estrutural das representações sociais. Revista Enfermagem UERJ, 10(2), 81-84.

Thiengo, M. A., Oliveira, D. C., \& Rodrigues, B. M. R. D. (2005). Representações sociais do HIV/aids entre adolescentes: Implicações para os cuidados de enfermagem. Revista da Escola de Enfermagem da USP, $39(1), 68-76$.

Trajman, A., Belo, M. T., Teixeira, E. G., Dantas, V. C. S., Salomão, F. M., \& Cunha, A. J. L. A. (2003). Knowledge about STD/AIDS and sexual behavior among high school students in Rio de Janeiro, Brazil. Cadernos de Saúde Pública, 19(1), 127-133.

Tura, L. F. R. (1998). Aids e estudantes: A estrutura das representações sociais. In D. Jodelet \& M. Madeira (Orgs.), Aids e representações sociais: A busca de sentidos (pp. 121-154). Natal: Ed. UFRN.

Vieira, K. F. L., Amaral, A. C. G., \& Saldanha, A. A. W. (2007). A vulnerabilidade à aids a partir das representações sociais de adolescentes. Jornada Internacional, 5; Conferência Brasileira Sobre Representações Sociais, 3, 1-10.

Wagner, W. (1998). Sociogênese e características das representações sociais. In A. S. P. Moreira \& D. C. Oliveira (Orgs.), Estudos interdisciplinares de representação social (pp. 3-25). Goiânia: AB.
Worobey, M., Gemmel, M., Teuwen, D. E., Haselkorn, T., Kunstman, K., Bunce, M., Muyembe, J. J., Kabongo, J. M., Kalengayi, R. M., Van Marck, E., Gilbert, M. T., \& Wolinsky, S. M. (2008). Direct evidence of extensive diversity of HIV-1 in Kinshasa by 1960. Nature, 455(7213), 661-664.

Jean Carlos Natividade é doutorando do Programa de Pós-graduação em Psicologia da Universidade Federal do Rio Grande do Sul. Brígido Vizeu Camargo é Professor Associado do Departamento de Psicologia da Universidade Federal de Santa Catarina.
Recebido: 09/09/2009

$1^{a}$ revisão: $18 / 01 / 2010$

$2^{a}$ revisão: 06/02/2010

Aceite final: 14/09/2010 